\title{
Evaluation of a nutrient-based diet quality index in UK young children and investigation into the diet quality of consumers of formula and infant foods
}

\author{
Eric $\bigcirc$ Verger ${ }^{1,2, *}$, Simone Eussen ${ }^{3}$ and Bridget $A$ Holmes ${ }^{4}$ \\ ${ }^{1}$ Institute of Cardiometabolism and Nutrition (ICAN), Assistance Publique-Hôpitaux de Paris, Pitié-Salpêtrière \\ Hospital, Nutrition Department, F-75013 Paris, France: ${ }^{2}$ Institut National de la Santé et de la Recherche Médicale \\ (INSERM), Research Unit S1 166 (UMR S1 166), Nutriomics Team, Paris, France: ${ }^{3}$ Nutricia Research, Utrecht, \\ The Netherlands: ${ }^{4}$ Danone Nutricia Research, Centre Daniel Carasso, Palaiseau, France
}

Submitted 31 March 2015: Final revision received 17 September 2015: Accepted 12 October 2015: First published online 2 November 2015

\begin{abstract}
Objective: To adapt and evaluate a nutrient-based diet quality index (PANDiet) for UK young children and to determine the nutritional adequacy of their diets according to consumption of young child formula (YCF) and commercial infant foods (CIF).

Design: Content and construct validity of the PANDiet were assessed by studying associations between the PANDiet and its components, energy intake, food intakes, and child and maternal characteristics. Four groups of children were defined according to their intake of YCF and CIF: (i) no consumption; (ii) consumption of YCF; (iii) consumption of CIF; and (iv) consumption of YCF and CIF. Child and maternal characteristics, PANDiet scores and food intakes of these four groups were compared.

Setting: Secondary analysis of data from the UK Diet and Nutrition Survey of Infants and Young Children (DNSIYC, 2011).

Subjects: Young children ( $n$ 1152) aged $12-18$ months.

Results: The PANDiet was adapted to the UK based on twenty-five nutrients. A lower PANDiet score was linked to lower intakes of YCF, CIF, vegetables and fruits. Determinants of having a lower score were being older, having siblings and having a younger mother with a lower educational level. Compared with children consuming neither YCF nor CIF, PANDiet scores were higher in children consuming CIF $(+1 \cdot 4)$, children consuming YCF $(+7 \cdot 2)$ and children consuming YCF and CIF $(+7 \cdot 8$; all $P<0 \cdot 001)$.

Conclusions: The PANDiet is a valid indicator of the nutrient adequacy of the diet of UK young children. Consuming CIF was not found to be associated with lower nutritional adequacy whereas consuming YCF was associated with higher nutritional adequacy.
\end{abstract}

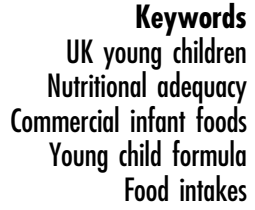

It is well recognized that the first 2 years of life are particularly important for health in later life and that adequate nutrition is essential to optimize health, physical and mental development, and reduce the risk of chronic disease $^{(1)}$. More specifically, between 6 and 24 months of age, children are switching from an exclusive milk diet to more adult-like meal and snack patterns which involve an increased variety of foods and nutrient compositions ${ }^{(2)}$. Children are also acquiring many self-feeding skills and experiences with food which further define their dietary patterns ${ }^{(3)}$. The UK Department of Health and the British Nutrition Foundation recommend that children aged
6-24 months get all the nutrients they need through acquiring the habit of eating a healthy and varied diet based mainly on foods that are usually consumed in the family ${ }^{(4,5)}$.

The potential contribution that young child formula (YCF; like growing-up milk and follow-on milk) and commercial infant foods (CIF; like meat-, fish- or cerealbased ready-to-eat dishes) make to a healthy diet among children aged 12 months and over remains unclear. According to the European Food Safety Authority and the UK Department of Health, YCF is one of several means to prevent the risk of inadequate status of $n-3$ PUFA, Fe and 
vitamin D in children older than 12 months, on the same level as fortified cow's milk, fortified cereals and cerealbased foods or supplements ${ }^{(4,6)}$. While the European Food Safety Authority specifies that currently marketed YCF contains more $n-3$ PUFA, Fe and vitamin D than cow's milk $^{(6)}$, the UK consumer association Which? reported that YCF contains less Ca and more sugar than cow's milk and is also more expensive ${ }^{(7)}$. The consumption of CIF and the contribution that CIF makes to the diet of young children are not well understood. The UK Department of Health suggests that while CIF seems to be useful on an occasional basis, it should not be consumed too frequently due to the high levels of salt and sugar ${ }^{(4)}$. A recent analysis of the UK market highlighted that CIF are predominantly sweet foods that provide little added value in terms of nutrient density ${ }^{(8)}$. In addition, literature concerning the contribution of YCF and CIF to the diet of young children is scarce and inconsistent ${ }^{(8-13)}$.

Diet quality indices are useful tools to provide an overall measure of the quality of the diet based on current nutrition knowledge ${ }^{(14)}$. Most existing indices for infants and young children are based on foods, food groups, dietary guidelines or food variety ${ }^{(15)}$. The uncertainty surrounding the contribution that YCF and CIF make to a healthy diet is also present in such indices, reflecting the difficulty in balancing the potential contribution from the different nutrients in these foods. For example, the Finnish Children Healthy Eating Index (FCHEI), developed for children aged 1-6 years, included CIF as a fair contributor to the intakes of vegetables, fruit, fish and fish dishes like any other contributors, such as home-made dishes ${ }^{(16)}$, whereas in the Complementary Feeding Utility Index (CFUI) developed for UK children aged 6 months, CIF were included as a food group for which the consumption should be moderated ${ }^{(17)}$. The PANDiet, a nutrient-based diet quality index, has been developed and previously validated in adult populations ${ }^{(18)}$. Since the PANDiet measures diet quality in terms of global nutrient adequacy rather than food intakes, it can be used to objectively measure diet quality and help to elucidate the ambiguity regarding the contribution that different food groups make to the nutrient adequacy of the diet.

The objectives of the present study were to: (i) adapt the PANDiet for UK young children aged 12-18 months; (ii) evaluate its content and construct validity; and (iii) determine the nutritional adequacy of the diet in groups of children according to their consumption of YCF and CIF.

\section{Materials and methods}

\section{Participants and data}

Data used in the current analysis came from the UK Diet and Nutrition Survey of Infants and Young Children (DNSIYC, 2011). The design, methodology and results of the DNSIYC have been described in full elsewhere ${ }^{(19)}$. Briefly, the DNSIYC was a descriptive cross-sectional national survey undertaken on a randomly selected sample of 2683 infants and young children aged 4-18 months living in private households in the UK. Parents were asked to keep a record of everything the infant or young child ate or drank over four consecutive days using a food diary. Portion sizes were recorded in household measures or using the weight indicated on packaged foods. Breast milk intake was calculated in grams based on the duration of each feed, at $10 \mathrm{~g} / \mathrm{min}$ with a maximum of $100 \mathrm{~g}$ per feed for young children aged 8-18 months ${ }^{(19)}$. Nutritional values for energy and nutrients came from the Department of Health's Nutrient Databank ${ }^{(20)}$. Mean individual intakes of foods and nutrients were calculated using the weighting scheme proposed by DNSIYC. Mean individual intakes of nutrients are from food sources only and do not include nutrient intakes from dietary supplements. The foods and drinks were classified into forty-five categories ${ }^{(19)}$ that have primarily been used as such for the current analysis.

In the current analysis, only the children aged 12-18 months were selected ( $n$ 1275). We first excluded those with missing information for analysed variables ( $n$ 99) and then removed those with extreme values in the energy intake distribution (respectively lower and higher than the 1 st and 99th percentiles, $n 24)^{(21)}$. This resulted in a final number of 1152 children ( $90 \%$ of the children aged 12-18 months) available for analysis.

\section{PANDiet for UK children aged 12-18 months}

The PANDiet aims to measure the overall diet quality of an individual through the probability of having an adequate nutrient intake. The construction and design of the PANDiet have been described in detail elsewhere ${ }^{(18)}$. Briefly, the PANDiet, which is made up of the Adequacy sub-score and the Moderation sub-score, relies on the calculation of the probability of adequacy for each available nutrient, ranging from 0 to 1 , where 1 represents a $100 \%$ probability that the intake is adequate according to a reference value. The calculation of the probability takes into account the number of days of dietary data, the mean intake and the day-to-day variability in intake, the nutrient reference value and inter-individual variability. The adequate intake of a nutrient was assumed to be the level likely to satisfy the nutrient requirements and unlikely to be excessive. Consequently, the Adequacy sub-score computed the probability of adequacy for nutrients for which the usual intake should be above a reference value (the higher the sub-score, the better the intake satisfies the nutrient requirements), and the Moderation sub-score computed the probability of adequacy for nutrients for which the usual intake should not exceed a reference value (the higher the sub-score, the less likely the intake is excessive). All the probabilities of adequacy within each sub-score were equally weighted. The PANDiet score is taken as the mean of the Adequacy and Moderation 
sub-scores and ranges from 0 to 100; the higher the score, the better the nutrient adequacy of the diet.

Based on the PANDiet that was developed originally, the current nutritional recommendations and the nutrients available in the Department of Health's Nutrient Databank, we selected twenty-five nutrients for inclusion in the PANDiet for UK children aged 12-18 months: protein, total carbohydrates, non-milk extrinsic sugars (NMES; which includes all sugars known as added sugars monosaccharides and disaccharides added to foods by the manufacturer, cook or consumer, and sugars naturally present in honey and syrups - and all sugars in fruit juices, plus $50 \%$ of the sugars in canned, stewed, dried or preserved fruits $\left.{ }^{(22)}\right)$, total fat, SFA, PUFA, thiamin, riboflavin, niacin, folate, vitamins $\mathrm{A}, \mathrm{B}_{6}, \mathrm{~B}_{12}, \mathrm{C}$ and $\mathrm{D}$, and minerals $\mathrm{Ca}$, $\mathrm{Mg}, \mathrm{Zn}, \mathrm{P}, \mathrm{K}, \mathrm{Fe}, \mathrm{Cu}, \mathrm{Se}$, iodine and $\mathrm{Na}$. For protein and micronutrients, we used the reference value and interindividual variability as described in the UK nutritional recommendations for children aged 12-36 months ${ }^{(23)}$. For other macronutrients, no UK nutritional recommendations for children aged less than 5 years have been developed. We therefore used the reference value as described in the European Food Safety Authority recommendations for children aged 12-36 months for total carbohydrates, total fat and PUFA ${ }^{(6)}$. In order to be as complete as possible, and because the Nordic recommendations for children aged 12-23 months and for adults and children from 2 years of age do not differ for added sugars and SFA ${ }^{(24)}$, we used the reference value and inter-individual variability as described in the UK nutritional recommendations for children aged more than 5 years for NMES and SFA. The set of reference values and inter-individual variability used for the PANDiet for UK children aged 12-18 months are shown in the online supplementary material, Supplemental Table 1.

\section{Evaluation of the content and construct validity of the PANDiet}

The strategy to evaluate the content and construct validity of the PANDiet has been described previously in the French and US adult populations ${ }^{(18)}$. In order to evaluate the content and construct validity of the PANDiet in this new population, we adopted the same strategy as used previously, but using variables linked to diet quality in UK young children. Recent reviews indicated that diet quality indices are significantly associated with various sociodemographic variables, including age, sex, race/ethnicity, income, parental education, socio-economic status, place of living and marital status of parents ${ }^{(15,25,26)}$. More specifically, Robinson et al. found that UK young children aged 6 and 12 months with healthier dietary patterns were more likely to be born first and their mothers were more likely to have a lower BMI, be better educated, be older and be a non-smoker ${ }^{(27)}$. Consistently, Golley et al. found that the mothers of UK young children aged 6 months with better diet quality - as measured by the CFUI score - were more likely to have a lower BMI, be better educated, be older, be a non-smoker, be married and belong to a higher social class ${ }^{(17)}$.

Accordingly, the adapted strategy to evaluate the content and construct validity consisted of: (i) investigating the correlations between the PANDiet score and its components; (ii) testing the absence of correlation between the PANDiet score and energy intake in order to verify that a higher score from the PANDiet was not automatically attributed to a higher-energy diet; (iii) evaluating the associations between the PANDiet score and food intakes; and (iv) evaluating the associations between the PANDiet score and child and maternal characteristics available in the DYNSIC data set. Child characteristics used included age, sex, being the first born ('yes' or 'no'), having siblings ('no', 'one' or 'two or more') and ethnicity (as defined in the DYNSIC survey, i.e. 'White', 'South Asian' or 'Other', where the group 'Other' represents children belonging to Black ethnic groups and children with parents belonging to mixed ethnic groups). Maternal characteristics used included age, BMI $\left(\mathrm{kg} / \mathrm{m}^{2}\right)$, being single ('yes' or 'no', defined as not living as a couple and never having been married), smoking status ('never and ex-smoker' or 'current smoker'), educational level ('degree level or above', 'qualification below degree level' or 'no qualifications') and social class ('managerial and professional', 'intermediate', 'routine and manual' or 'not classifiable').

\section{Classification according to intakes of young child formula and commercial infant foods}

The 1152 young children aged 12-18 months were classified into four groups according to their consumption of YCF and CIF, where consumption was defined as an intake higher than $0 \mathrm{~g}$. Accordingly, the groups $\mathrm{YCF}^{-} / \mathrm{CIF}^{-}$ (no consumption of either YCF or CIF), $\mathrm{YCF}^{-} / \mathrm{CIF}^{+}$(no consumption of YCF but consumption of $\mathrm{CIF}$ ), $\mathrm{YCF}^{+} / \mathrm{CIF}^{-}$ (consumption of YCF but no consumption of CIF) and $\mathrm{YCF}^{+} / \mathrm{CIF}^{+}$(consumption of YCF and $\mathrm{CIF}$ ) were defined.

\section{Statistical analyses}

All analyses were performed using the statistical software package SAS version 9.3. Continuous variables are presented as means and their standard errors, and categorical variables are presented as percentages. Descriptive statistics of the PANDiet components, sub-scores, energy intake and food intakes were assessed across quartiles of PANDiet scores. Spearman correlation coefficients were used to examine the correlations between the total PANDiet score and the PANDiet components, sub-scores, energy intake and food intakes. The associations between the lowest PANDiet quartile (outcome variable) and child and maternal characteristics (predictor variables) were explored using logistic regression. All the child and maternal characteristics were included in the model simultaneously and the backwards elimination method was used to identify the independent predictor variables of the associations. The results are reported as adjusted odds ratios and 95\% confidence 
intervals. Descriptive statistics of the child and maternal characteristics, PANDiet components, sub-scores, scores, energy intake and food intakes were assessed across the four groups defined according to their consumption of YCF and CIF. The $\chi^{2}$ and Wilcoxon rank-sum tests were used to compare child and maternal characteristics across the four groups. When $\chi^{2}$ identified a significant effect, cell $\chi^{2}$ values were used to understand the relative contribution to the overall $\chi^{2}$. When Wilcoxon rank-sum identified a significant effect, a Dwass, Steel and Critchlow-Fligner (DSCF) multiple comparison analysis was used. ANCOVA was used compare the mean PANDiet components, sub-scores, scores, energy intake and food intakes across the four groups, adjusted for age and energy intake. Box-Cox transformation was used when residuals were not normally distributed. When ANCOVA identified a significant effect, pair-wise post hoc tests were used. The level of significance used was $P<0.05$ unless specified otherwise. Bonferroni correction was used for the adjustment of the PANDiet components, sub-scores, scores, energy, nutrient and food analysis.

\section{Results}

\section{Evaluation of the content and construct validity of the PANDiet}

Child and maternal characteristics of the studied sample are displayed in Table 1. The mean PANDiet score was 70.2 (SE 0.2) and the PANDiet scores ranged from 48.6 to 92.7 with an approximately normal distribution (skewness $=0.02$ and kurtosis $=0 \cdot 18$ ). The mean Adequacy sub-score was almost twice the mean Moderation sub-score $(86.5(\operatorname{se} 0.2) v .54 .0(\operatorname{se} 0.3))$, indicating that recommendations linked to avoiding excessive nutrient intakes were less well respected in this population. Notably, the mean probabilities for avoiding excessive $\mathrm{Na}$ and SFA intakes were very low: 0.13 (se 0.01) and 0.12 (SE 0.01), respectively. Descriptive statistics of the PANDiet components, sub-scores and energy intake across quartiles of PANDiet scores are displayed in the online supplementary material, Supplemental Table 2. The Spearman correlations between the PANDiet score and PANDiet components were as expected compared with the previous version of the PANDiet, and the Spearman correlation between the PANDiet score and energy intake was very weak $(\rho=0.063, P=0.032)$.

Figure 1 presents the results for the PANDiet score according to twelve food groups identified as having the highest inverse and positive Spearman correlations with the PANDiet score. The lower the PANDiet score, the higher the intakes of whole milk, sugar, preserves and confectionery, burgers, kebabs, sausages, meat pies and pastries, biscuits and soft drinks (low-calorie and not lowcalorie) and the lower the intakes of vegetables, fruits, YCF and CIF (snacks - sweet and savoury, meat- and fish-based products and dishes, cereal-based foods and dishes).
Table 1 Child and maternal characteristics of the sample of young children ( $n$ 1152) aged 12-18 months, UK Diet and Nutrition Survey of Infants and Young Children (DNSIYC, 2011)

\begin{tabular}{|c|c|c|c|}
\hline & $\%$ & Mean & SE \\
\hline \multicolumn{4}{|l|}{ Child characteristics } \\
\hline PANDiet score & & $70 \cdot 2$ & 0.2 \\
\hline Adequacy sub-score & & $86 \cdot 5$ & 0.2 \\
\hline Moderation sub-score & & $54 \cdot 0$ & 0.3 \\
\hline Age (months) & & $14 \cdot 3$ & 0.05 \\
\hline Sex-boys & $50 \cdot 6$ & & \\
\hline First child & $49 \cdot 3$ & & \\
\hline \multicolumn{4}{|l|}{ Siblings } \\
\hline None & $47 \cdot 4$ & & \\
\hline One & 31.4 & & \\
\hline Two or more & $21 \cdot 2$ & & \\
\hline \multicolumn{4}{|l|}{ Ethnicity } \\
\hline White & $81 \cdot 8$ & & \\
\hline South Asian & 8.0 & & \\
\hline Other & $10 \cdot 2$ & & \\
\hline \multicolumn{4}{|l|}{ Maternal characteristics } \\
\hline Age (years) & & $30 \cdot 2$ & 0.17 \\
\hline BMI $\left(\mathrm{kg} / \mathrm{m}^{2}\right)$ & & $26 \cdot 3$ & 0.15 \\
\hline Single & $15 \cdot 2$ & & \\
\hline Smoking status - smoker & 21.7 & & \\
\hline \multicolumn{4}{|l|}{ Educational level } \\
\hline Degree level or above & $32 \cdot 3$ & & \\
\hline Qualification below degree level & $58 \cdot 0$ & & \\
\hline No qualifications & $9 \cdot 7$ & & \\
\hline \multicolumn{4}{|l|}{ Social class } \\
\hline Managerial and professional & $38 \cdot 7$ & & \\
\hline Intermediate & $19 \cdot 3$ & & \\
\hline Routine and manual & $35 \cdot 1$ & & \\
\hline Not classifiable & 6.9 & & \\
\hline
\end{tabular}

Table 2 Associations of child and maternal characteristics with the first quartile of the PANDiet ${ }^{*}$ among young children $(n$ 1152) aged 12-18 months, UK Diet and Nutrition Survey of Infants and Young Children (DNSIYC, 2011)

\begin{tabular}{|c|c|c|c|}
\hline & OR & $95 \% \mathrm{Cl}$ & $P$ value \\
\hline \multicolumn{4}{|l|}{ Child characteristics } \\
\hline Age (months) & $1 \cdot 148$ & $1.078,1.223$ & $<0.001$ \\
\hline Siblings & & & 0.021 \\
\hline None & 1.000 & Ref. & \\
\hline One & 1.381 & $1 \cdot 080,1 \cdot 765$ & \\
\hline Two or more & 1.343 & $1.002,1.800$ & \\
\hline Ethnicity & & & 0.003 \\
\hline White & 1.000 & Ref. & \\
\hline South Asian & 0.502 & $0.336,0.752$ & \\
\hline Other & 0.814 & $0.574,1.154$ & \\
\hline \multicolumn{4}{|l|}{ Maternal characteristics } \\
\hline Age (years) & 0.970 & $0.951,0.990$ & 0.003 \\
\hline Educational level & & & 0.002 \\
\hline Degree level or above & 1.000 & Ref. & \\
\hline Qualification below degree level & 1.404 & $1.090,1.808$ & \\
\hline No qualifications & $2 \cdot 030$ & $1.340,3.076$ & \\
\hline
\end{tabular}

Ref., referent category.

${ }^{*}$ Results of logistic regression using the backwards elimination method are reported as adjusted odds ratio and $95 \%$ confidence intervals. All variables shown in the table, characteristics of the child (sex and being the first born) and characteristics of the mother (BMI, being single, smoking and social class) were included in the model simultaneously.

In the multivariate logistic regression analysis, the determinants of belonging to the lowest PANDiet quartile (the group with the lowest diet quality) were being older, having one or more siblings, having a younger mother and 


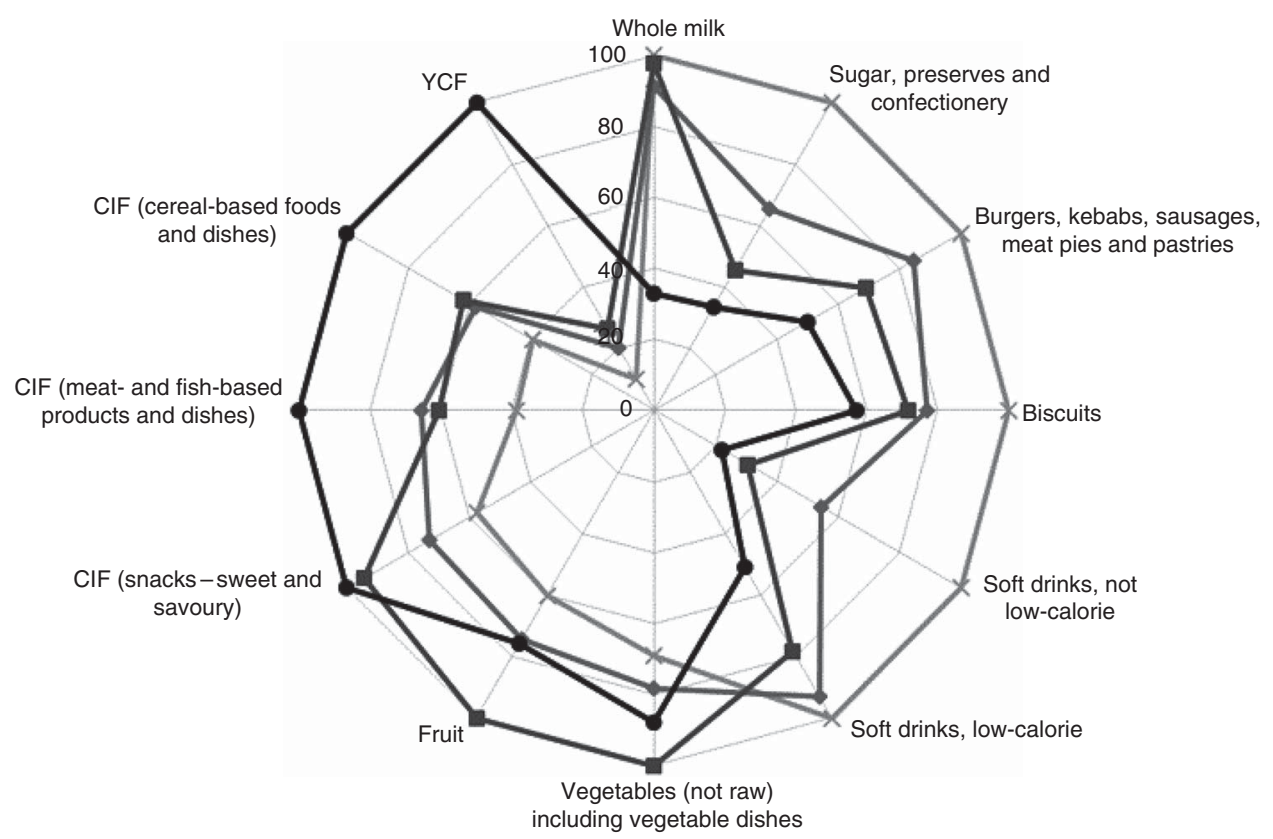

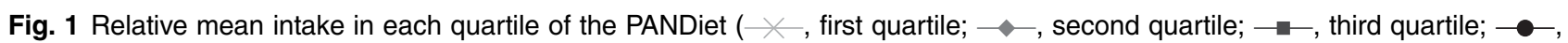
fourth quartile), shown as a percentage compared with the highest observed mean intake across the quartiles for twelve selected food groups*, among young children ( $n$ 1152) aged 12-18 months, UK Diet and Nutrition Survey of Infants and Young Children (DNSIYC, 2011). *The higher the PANDiet score, the better the nutrient adequacy of the diet. The twelve food groups shown are those with the highest inverse and positive Spearman correlation with the PANDiet score (YCF, young child formula; CIF, commercial infant foods)

having a mother with a lower educational level (Table 2). We also found a lower percentage of South Asian children in the lowest PANDiet quartile compared with the other quartiles.

\section{Nutrient adequacy according to intakes of young child formula and commercial infant foods}

Twenty-seven per cent of the young children were classified in the $\mathrm{YCF}^{-} / \mathrm{CIF}^{-}$group ( $n$ 313), $36 \%$ in the $\mathrm{YCF}^{-} /$ $\mathrm{CIF}^{+}$group ( $n$ 418), $8 \%$ in the $\mathrm{YCF}^{+} / \mathrm{CIF}^{-}$group $(n 93)$ and $29 \%$ in the $\mathrm{YCF}^{+} / \mathrm{CIF}^{+}$group ( $n$ 328). Child and maternal characteristics according to consumption of YCF and CIF are displayed in the online supplementary material, Supplemental Table 3. Compared with other groups, the children in the $\mathrm{YCF}^{-} / \mathrm{CIF}^{-}$group were older and had a younger mother. Most of the relative contribution to the overall $\chi^{2}$ came from the $\mathrm{YCF}^{-} / \mathrm{CIF}^{-}$group, with less first-born children, more children with two or more siblings, more children with a single mother, more children with a mother who smoked, and less children with a mother with a higher educational level and a higher social class (cell $\chi^{2}$ values analysis, data not shown).

Descriptive statistics of the PANDiet components, sub-scores and energy intake according to the consumption of YCF and CIF are displayed in Table 3. PANDiet scores were significantly different across the four groups but energy intakes did not differ. Compared with the $\mathrm{YCF}^{-} / \mathrm{CIF}^{-}$group, children in the $\mathrm{YCF}^{-} / \mathrm{CIF}^{+}$group had a PANDiet score 1.4 points higher, children in the $\mathrm{YCF}^{+} / \mathrm{CIF}^{-}$ group had a PANDiet score 7.2 points higher and children in the $\mathrm{YCF}^{+} / \mathrm{CIF}^{+}$group had a PANDiet score 7.8 points higher. Compared with other groups, the children in the $\mathrm{YCF}^{+} / \mathrm{CIF}^{-}$and $\mathrm{YCF}^{+} / \mathrm{CIF}^{+}$groups had better nutrient adequacy for SFA, PUFA, vitamin D, Zn, Fe and $\mathrm{Cu}$. Descriptive statistics of the nutrient intakes according to the consumption of YCF and CIF are displayed in the online supplementary material, Supplemental Table 4 .

Mean food intakes according to the consumption of YCF and CIF are displayed in Table 4. Compared with the $\mathrm{YCF}^{-} / \mathrm{CIF}^{-}$group, the children in the $\mathrm{YCF}^{+} \mathrm{CIF}^{+}$group consumed significantly more dairy products excluding cheese, YCF and CIF, and less of several food groups including white bread, whole milk, snacks, chips and soft drinks. Compared with the $\mathrm{YCF}^{-} / \mathrm{CIF}^{-}$group, the children in the $\mathrm{YCF}^{-} / \mathrm{CIF}^{+}$group consumed significantly more whole milk, dairy products excluding cheese and CIF (except dairy-based CIF), and less of other including white bread, snacks, chips and soft drinks. Compared with the $\mathrm{YCF}^{-} / \mathrm{CIF}^{-}$group, the children in the $\mathrm{YCF}^{+} / \mathrm{CIF}^{-}$group consumed significantly more YCF and less breakfast cereals, whole and semi-skimmed milk, and soft drinks (not low-calorie). The breast milk intake was significantly lower in the $\mathrm{YCF}^{+} / \mathrm{CIF}^{+}$group compared with the $\mathrm{YCF}^{-} / \mathrm{CIF}^{-}$and $\mathrm{YCF}^{-} / \mathrm{CIF}^{+}$groups although intakes across all groups were very low, as expected for this age range: $7.7 \%$ of the children in the $\mathrm{YCF}^{-} / \mathrm{CIF}^{-}$group were still breast-feeding, $12.0 \%$ in the $\mathrm{YCF}^{-} / \mathrm{CIF}^{+}$group, $4.3 \%$ in the $\mathrm{YCF}^{+} / \mathrm{CIF}^{-}$group and $3.7 \%$ in the $\mathrm{YCF}^{+} / \mathrm{CIF}^{+}$group. 
Table 3 Energy intake, PANDiet scores and sub-scores and individual components according to YCF and CIF consumer groups ${ }^{\star}$ among young children ( $n$ 1152) aged 12-18 months, UK Diet and Nutrition Survey of Infants and Young Children (DNSIYC, 2011)

\begin{tabular}{|c|c|c|c|c|c|c|c|c|c|}
\hline & \multicolumn{2}{|c|}{$\mathrm{YCF}^{-} / \mathrm{CIF}^{-}(n$ 313) } & \multicolumn{2}{|c|}{$\mathrm{YCF}^{-} / \mathrm{CIF}^{+}(n 418)$} & \multicolumn{2}{|c|}{$\mathrm{YCF}^{+} / \mathrm{CIF}^{-}(n 93)$} & \multicolumn{2}{|c|}{$\mathrm{YCF}^{+} / \mathrm{CIF}^{+}(n$ 328) } & \multirow[b]{2}{*}{ ANCOVA $P$ value } \\
\hline & Mean & SE & Mean & SE & Mean & SE & Mean & SE & \\
\hline Energy intake $(\mathrm{kJ} / \mathrm{d})$ & 4140 & 47 & 4108 & 39 & 3933 & 80 & 3975 & 43 & 0.013 \\
\hline Energy intake $(\mathrm{kcal} / \mathrm{d})$ & 984 & 11 & 976 & 9 & 935 & 19 & 944 & 10 & 0.013 \\
\hline PANDiet score & $66 \cdot 9^{a}$ & 0.3 & $68 \cdot 3^{b}$ & 0.3 & $74 \cdot 1^{\mathrm{c}}$ & 0.6 & $74.7^{c}$ & 0.3 & $<0.001$ \\
\hline Moderation sub-score & $51 \cdot 8^{a}$ & 0.6 & $52 \cdot 9^{a}$ & 0.5 & $56 \cdot 0^{\mathrm{b}}$ & 1.0 & $56 \cdot 9^{\mathrm{b}}$ & 0.5 & $<0.001$ \\
\hline Total carbohydrates & $0.91^{a}$ & 0.01 & $0.90^{\mathrm{a}}$ & 0.01 & $0.91^{a}$ & 0.03 & $0.78^{\mathrm{b}}$ & 0.01 & $<0.001$ \\
\hline Total fat & $0.73^{a}$ & 0.02 & $0.81^{\mathrm{b}}$ & 0.01 & $0.80^{a, b}$ & 0.03 & $0.89^{c}$ & 0.02 & $<0.001$ \\
\hline SFA & $0.09^{a}$ & 0.01 & $0.06^{\mathrm{a}}$ & 0.01 & $0.17^{b}$ & 0.02 & $0.21^{\mathrm{b}}$ & 0.01 & $<0.001$ \\
\hline NMES & 0.78 & 0.02 & 0.77 & 0.02 & 0.82 & 0.03 & 0.75 & 0.02 & 0.300 \\
\hline $\mathrm{Na}$ & $0.09^{a}$ & 0.01 & $0 \cdot 10^{\mathrm{b}}$ & 0.01 & $0 \cdot 10^{\mathrm{b}}$ & 0.02 & $0.22^{c}$ & 0.01 & $<0.001$ \\
\hline Adequacy sub-score & $82 \cdot 1^{\mathrm{a}}$ & 0.3 & $83 \cdot 7^{b}$ & 0.2 & $92 \cdot 3^{\mathrm{c}}$ & 0.5 & $92 \cdot 4^{\mathrm{C}}$ & 0.2 & $<0.001$ \\
\hline Protein & 1.00 & 0.00 & 1.00 & 0.00 & 1.00 & 0.00 & 1.00 & 0.00 & 0.076 \\
\hline Total carbohydrates & $0.77^{a}$ & 0.01 & $0.85^{\mathrm{b}}$ & 0.01 & $0.91^{\mathrm{b}, \mathrm{c}}$ & 0.03 & $0.96^{c}$ & 0.01 & $<0.001$ \\
\hline Total fat & $0.61^{a}$ & 0.02 & $0.54^{a}$ & 0.02 & $0.56^{\mathrm{a}}$ & 0.04 & $0.40^{\mathrm{b}}$ & 0.02 & $<0.001$ \\
\hline PUFA & $0.38^{a}$ & 0.02 & $0.26^{\mathrm{b}}$ & 0.01 & $0.73^{\mathrm{C}}$ & 0.03 & $0.65^{c}$ & 0.02 & $<0.001$ \\
\hline Vitamin A & $0.75^{\mathrm{a}}$ & 0.01 & $0.88^{b}$ & 0.01 & $0.92^{b}$ & 0.02 & $0.97^{\mathrm{C}}$ & 0.01 & $<0.001$ \\
\hline Thiamin & $1.00^{a}$ & 0.00 & $1.00^{b}$ & 0.00 & $1.00^{b}$ & 0.00 & $1.00^{b}$ & 0.00 & $<0.001$ \\
\hline Riboflavin & $0.96^{a}$ & 0.01 & $0.99^{b}$ & 0.00 & $0.98^{a, b}$ & 0.01 & $0.99^{b}$ & 0.01 & $<0.001$ \\
\hline Niacin & $1.00^{a}$ & 0.00 & $1.00^{a}$ & 0.00 & $1.00^{\mathrm{b}}$ & 0.00 & $1.00^{\mathrm{a}}$ & 0.00 & $<0.001$ \\
\hline Vitamin $\mathrm{B}_{6}$ & $0.98^{\mathrm{a}}$ & 0.00 & $0.99^{a}$ & 0.00 & $0.97^{a, b}$ & 0.01 & $0.96^{b}$ & 0.00 & $<0.001$ \\
\hline Folate & 0.99 & 0.00 & 0.99 & 0.00 & 0.99 & 0.00 & 1.00 & 0.00 & 0.058 \\
\hline Vitamin $B_{12}$ & 0.99 & 0.00 & 1.00 & 0.00 & 1.00 & 0.00 & 1.00 & 0.00 & 0.017 \\
\hline Vitamin C & $0.87^{a}$ & 0.01 & $0.92^{\mathrm{b}}$ & 0.01 & $0.99^{c}$ & 0.01 & $1.00^{c}$ & 0.01 & $<0.001$ \\
\hline Vitamin D & $0.00^{a}$ & 0.01 & $0.02^{b}$ & 0.01 & $0.63^{c}$ & 0.02 & $0.67^{c}$ & 0.01 & $<0.001$ \\
\hline $\mathrm{Ca}$ & $0.97^{\mathrm{a}}$ & 0.00 & $0.99^{b}$ & 0.00 & $0.99^{a, b}$ & 0.01 & $0.99^{b}$ & 0.00 & $<0.001$ \\
\hline $\mathrm{Mg}$ & $0.99^{a, b}$ & 0.00 & $1.00^{b}$ & 0.00 & $0.98^{\mathrm{a}}$ & 0.00 & $1.00^{b}$ & 0.00 & 0.002 \\
\hline $\mathrm{Zn}$ & $0.74^{a}$ & 0.01 & $0.79^{b}$ & 0.01 & $0.92^{C}$ & 0.02 & $0.94^{c}$ & 0.01 & $<0.001$ \\
\hline $\mathrm{P}$ & 1.00 & 0.00 & 1.00 & 0.00 & 1.00 & 0.00 & 1.00 & 0.00 & 0.749 \\
\hline $\mathrm{K}$ & $0.99^{a, b}$ & 0.00 & $1.00^{b}$ & 0.00 & $0.98^{a}$ & 0.00 & $0.99^{a, b}$ & 0.00 & $<0.001$ \\
\hline $\mathrm{Fe}$ & $0.40^{\mathrm{a}}$ & 0.02 & $0.47^{\mathrm{b}}$ & 0.01 & $0.90^{c}$ & 0.03 & $0.92^{c}$ & 0.02 & $<0.001$ \\
\hline $\mathrm{Cu}$ & $0.78^{a}$ & 0.01 & $0.82^{a}$ & 0.01 & $0.93^{b}$ & 0.02 & $0.97^{b}$ & 0.01 & $<0.001$ \\
\hline $\mathrm{Se}$ & $0.94^{a}$ & 0.01 & $0.93^{a}$ & 0.01 & $0.98^{\mathrm{b}}$ & 0.01 & $0.96^{b}$ & 0.01 & $<0.001$ \\
\hline lodine & $0.96^{a, b}$ & 0.01 & $0.98^{\mathrm{C}}$ & 0.01 & $0.94^{a}$ & 0.01 & $0.97^{b, c}$ & 0.01 & $<0.001$ \\
\hline
\end{tabular}

YCF, young child formula; CIF, commercial infant foods; NMES, non-milk extrinsic sugars.

$\mathrm{YCF}^{-} / \mathrm{CIF}^{-}$, no consumption of either $\mathrm{YCF}$ or $\mathrm{CIF}$; $\mathrm{YCF}^{-} / \mathrm{CIF}^{+}$, no consumption of $\mathrm{YCF}$ but consumption of $\mathrm{CIF}$; $\mathrm{YCF}{ }^{+} / \mathrm{CIF}^{-}$, consumption of $\mathrm{YCF}$ but no consumption of CIF; $\mathrm{YCF}^{+} / \mathrm{CIF}^{+}$, consumption of YCF and $\mathrm{CIF}$.

$a, b, c$ Mean values within a row with unlike superscript letters were significantly different as tested by pair-wise post hoc comparisons, when ANCOVA identified a significant effect with Bonferroni correction $(P \leq 0.002)$.

${ }^{*}$ Adjusted for age and energy intake. All the PANDiet components are expressed in absolute values ranging from 0 to 1 , where 1 represents a $100 \%$ probability that the intake is adequate according to a reference value. PANDiet score and the sub-scores are expressed in absolute values ranging from 0 to 100 ; the higher the score and sub-scores, the better the nutrient adequacy of the diet.

The intakes of vegetables, fruit, fish and water were not significantly different between the four groups.

\section{Discussion}

In the present study we adapted the PANDiet for UK young children aged 12-18 months and applied a fourstage strategy to evaluate the content and construct validity, which provided strong evidence suggesting that the PANDiet is a useful tool to assess diet quality in this population. Using this index, we evaluated the nutritional adequacy of the diet in groups of young children according to their YCF and CIF consumption. We found that the PANDiet scores were significantly higher in the groups consuming YCF or consuming YCF and CIF, mainly supported by a better probability of adequacy for SFA, PUFA, vitamin D, Zn, Fe and Cu. We found differences in terms of dietary pattern across the four groups, but vegetable, fruit, fish and water intakes did not differ significantly.

\section{Nutritional adequacy of UK young children}

Our results are coherent with those of the DNSIYC study which showed that the mean daily intakes of key vitamins and minerals were above or close to the recommended nutrient intake (RNI) and the mean daily $\mathrm{Na}$ intake was $181 \%$ of the $\mathrm{RNI}^{(19)}$. In our study, we indeed found that the average Adequacy sub-score was high (86.5 (SE 0.2)) and the $\mathrm{Na}$ intake was excessive as demonstrated by a very low probability of adequacy for $\mathrm{Na}(0 \cdot 13$ (SE 0.01)). Results of the DNSIYC study also showed that mean daily intakes of $\mathrm{Fe}$ and vitamin $\mathrm{D}$ were respectively moderately or far below the RNI. In our study, we found that the probability of adequacy for Fe was low for children in the first quartile $(0.33$ ( $\operatorname{se} 0.02))$ of the PANDiet and the 
Table 4 Daily food intakes in grams according to YCF and CIF consumer groups ${ }^{*}$ among young children $(n 1152)$ aged $12-18$ months, UK Diet and Nutrition Survey of Infants and Young Children (DNSIYC, 2011)

\begin{tabular}{|c|c|c|c|c|c|c|c|c|c|}
\hline & \multicolumn{2}{|c|}{$\begin{array}{c}\mathrm{YCF}^{-} / \mathrm{CIF}^{-} \\
(n 313) \\
\end{array}$} & \multicolumn{2}{|c|}{$\begin{array}{c}\mathrm{YCF}^{-} / \mathrm{CIF}^{+} \\
(n 418)\end{array}$} & \multicolumn{2}{|c|}{$\begin{array}{l}\mathrm{YCF}^{+} / \mathrm{CIF}^{-} \\
\quad(n 93)\end{array}$} & \multicolumn{2}{|c|}{$\begin{array}{l}\mathrm{YCF}^{+} / \mathrm{CIF}^{+} \\
(n 328)\end{array}$} & \multirow[b]{2}{*}{ ANCOVA $P$ value } \\
\hline & Mean & SE & Mean & $\mathrm{SE}$ & Mean & SE & Mean & SE & \\
\hline Pasta, rice, pizza and other cereals & 43.3 & $2 \cdot 4$ & $36 \cdot 1$ & $2 \cdot 0$ & $41 \cdot 3$ & $4 \cdot 3$ & $36 \cdot 0$ & $2 \cdot 3$ & 0.079 \\
\hline White bread & $20 \cdot 4^{\mathrm{a}}$ & 1.0 & $14 \cdot 6^{\mathrm{b}}$ & 0.9 & $18 \cdot 9^{\mathrm{a}, \mathrm{b}}$ & 1.8 & $12 \cdot 8^{\mathrm{b}, \mathrm{c}}$ & 1.0 & $<0.001$ \\
\hline Wholemeal bread & $5 \cdot 3$ & 0.7 & $6 \cdot 1$ & 0.6 & $2 \cdot 3$ & $1 \cdot 2$ & 5.4 & 0.6 & 0.041 \\
\hline Other breads & $5 \cdot 8$ & 0.7 & 6.9 & 0.6 & 4.4 & 1.2 & 4.7 & 0.6 & 0.050 \\
\hline Breakfast cereals & $23 \cdot 4^{a}$ & 1.6 & $19 \cdot 5^{\mathrm{a}, \mathrm{b}}$ & 1.4 & $13 \cdot 7^{\mathrm{b}, \mathrm{c}}$ & 2.8 & $13 \cdot 7^{\mathrm{C}}$ & 1.5 & $<0.001$ \\
\hline Biscuits & $7 \cdot 9^{a}$ & 0.4 & $6 \cdot 6^{\mathrm{a}}$ & 0.4 & $6 \cdot 1^{\mathrm{a}, \mathrm{b}}$ & 0.7 & $5 \cdot 1^{b}$ & 0.4 & $<0.001$ \\
\hline Buns, cakes, pastries and fruit pies & $5 \cdot 3$ & 0.5 & $5 \cdot 8$ & 0.4 & 4.6 & 0.9 & $3 \cdot 7$ & 0.5 & 0.015 \\
\hline Puddings & $10 \cdot 8$ & 1.4 & $13 \cdot 4$ & $1 \cdot 2$ & 11.4 & $2 \cdot 4$ & $11 \cdot 0$ & $1 \cdot 3$ & 0.428 \\
\hline Whole milk & $331^{\mathrm{a}}$ & 11 & $367^{\mathrm{b}}$ & 9 & $97^{\mathrm{C}}$ & 19 & $96^{\mathrm{C}}$ & 10 & $<0.001$ \\
\hline Semi-skimmed milk & $44 \cdot 3^{a}$ & $5 \cdot 6$ & $20 \cdot 9^{\mathrm{b}}$ & $4 \cdot 7$ & $16 \cdot 3^{\mathrm{b}, \mathrm{c}}$ & 9.9 & $4 \cdot 0^{\mathrm{c}}$ & $5 \cdot 3$ & $<0.001$ \\
\hline Other milk and cream & 7.5 & $2 \cdot 6$ & 8.4 & $2 \cdot 2$ & $2 \cdot 4$ & 4.6 & $2 \cdot 9$ & 2.5 & 0.306 \\
\hline Cheese & $7 \cdot 0$ & 0.5 & $7 \cdot 1$ & 0.4 & 7.5 & 0.9 & 7.5 & 0.5 & 0.892 \\
\hline Yoghurt, fromage frais and other dairy desserts & $45 \cdot 4^{a}$ & $2 \cdot 3$ & $55 \cdot 1^{\mathrm{b}}$ & 1.9 & $34 \cdot 3^{a}$ & 4.0 & $54 \cdot 5^{\mathrm{b}}$ & $2 \cdot 2$ & $<0.001$ \\
\hline Ice cream & $2 \cdot 3$ & 0.3 & $2 \cdot 3$ & 0.3 & 1.6 & 0.6 & 1.4 & 0.3 & 0.164 \\
\hline Eggs and egg dishes & $8 \cdot 2$ & 0.7 & $7 \cdot 7$ & 0.6 & $8 \cdot 1$ & 1.3 & $7 \cdot 6$ & 0.7 & 0.952 \\
\hline Butter & 1.0 & 0.2 & 1.7 & 0.2 & 0.8 & 0.3 & $1 \cdot 8$ & 0.2 & 0.002 \\
\hline Reduced- and low-fat spreads & $3 \cdot 1^{\mathrm{a}}$ & 0.2 & $2 \cdot 4^{\mathrm{b}}$ & 0.2 & $2 \cdot 6^{\mathrm{a}, \mathrm{b}}$ & 0.3 & $1.9^{\mathrm{b}}$ & 0.2 & $<0.001$ \\
\hline Red meat and dishes (bacon, ham, beef, veal, lamb, pork) & $24 \cdot 4$ & $2 \cdot 0$ & $25 \cdot 6$ & 1.7 & $29 \cdot 8$ & 3.5 & $26 \cdot 8$ & 1.9 & 0.570 \\
\hline White meat (chicken, turkey) & $16 \cdot 9$ & 1.4 & $15 \cdot 8$ & 1.2 & $19 \cdot 3$ & $2 \cdot 6$ & 13.8 & 1.4 & 0.208 \\
\hline Burgers, kebabs, sausages, meat pies and pastries & $14 \cdot 1^{a}$ & 0.9 & $7 \cdot 8^{\mathrm{b}}$ & 0.8 & $11 \cdot 3^{\mathrm{a}, \mathrm{b}}$ & 1.6 & $5 \cdot 6^{\mathrm{C}}$ & 0.9 & $<0.001$ \\
\hline Other (including liver and liver dishes and offal) & 0.06 & 0.05 & 0.10 & 0.04 & 0.09 & 0.09 & 0.07 & 0.05 & 0.960 \\
\hline Fish and fish dishes & 11.6 & 1.0 & $12 \cdot 1$ & 0.9 & 11.6 & 1.8 & $12 \cdot 4$ & 1.0 & 0.944 \\
\hline Salad and other raw vegetables & $5 \cdot 0$ & 0.6 & $4 \cdot 1$ & 0.5 & 4.0 & 1.0 & $3 \cdot 3$ & 0.5 & 0.236 \\
\hline Vegetables (not raw) including vegetable dishes & $41 \cdot 1$ & $2 \cdot 2$ & $40 \cdot 0$ & 1.8 & 39.4 & 3.8 & 37.5 & $2 \cdot 1$ & 0.684 \\
\hline Chips, fried and roast potatoes and potato products & $15 \cdot 0^{a}$ & 0.8 & $8 \cdot 1^{\mathrm{b}}$ & 0.7 & $13 \cdot 4^{\mathrm{a}}$ & 1.5 & $6 \cdot 3^{\mathrm{b}}$ & 0.8 & $<0.001$ \\
\hline Other potatoes, potato salads and dishes & $24 \cdot 8^{\mathrm{a}}$ & 1.6 & $19 \cdot 0^{\mathrm{b}}$ & 1.3 & $28 \cdot 6^{\mathrm{b}}$ & $2 \cdot 8$ & $18 \cdot 2^{\mathrm{b}}$ & 1.5 & $<0.001$ \\
\hline Savoury snacks & $3 \cdot 4^{a}$ & 0.2 & $1 \cdot 7^{\mathrm{b}}$ & 0.2 & $2 \cdot 6^{\mathrm{a}, \mathrm{b}}$ & 0.4 & $1.6^{\mathrm{b}}$ & 0.2 & $<0.001$ \\
\hline Fruit & 74.7 & 3.5 & 81.9 & $2 \cdot 9$ & $67 \cdot 1$ & $6 \cdot 1$ & 73.1 & $3 \cdot 3$ & 0.074 \\
\hline Sugar, preserves and confectionery & $5 \cdot 8^{a}$ & 0.4 & $3 \cdot 8^{\mathrm{b}}$ & 0.3 & $5 \cdot 9^{a}$ & 0.7 & $3 \cdot 8^{\mathrm{b}}$ & 0.4 & $<0.001$ \\
\hline Juices, including smoothies & $14 \cdot 2$ & $2 \cdot 1$ & $12 \cdot 2$ & $1 \cdot 8$ & $11 \cdot 3$ & 3.8 & 13.5 & $2 \cdot 0$ & 0.850 \\
\hline Soft drinks, not low-calorie & $74 \cdot 3^{\mathrm{a}}$ & $6 \cdot 8$ & $35 \cdot 6^{\mathrm{b}}$ & $5 \cdot 8$ & $26 \cdot 9^{\mathrm{b}}$ & 11.9 & $22 \cdot 5^{\mathrm{b}}$ & $6 \cdot 4$ & $<0.001$ \\
\hline Soft drinks, low-calorie & $130 \cdot 7^{\mathrm{a}}$ & $9 \cdot 3$ & $76 \cdot 1^{\mathrm{b}}$ & $7 \cdot 9$ & $90 \cdot 4^{a, b}$ & $16 \cdot 4$ & $44 \cdot 4^{b}$ & 8.8 & $<0.001$ \\
\hline Tea and coffee & $6 \cdot 0$ & $1 \cdot 1$ & $1 \cdot 7$ & 1.0 & 8.4 & $2 \cdot 0$ & $3 \cdot 8$ & 1.1 & 0.003 \\
\hline Water & 100 & 8 & 118 & 6 & 113 & 13 & 120 & 7 & 0.227 \\
\hline Soup & $16 \cdot 4$ & $2 \cdot 4$ & 11.5 & $2 \cdot 0$ & $16 \cdot 0$ & $4 \cdot 2$ & $17 \cdot 0$ & $2 \cdot 2$ & 0.232 \\
\hline Savoury sauces, pickles, gravies and condiments & $6 \cdot 8$ & 0.7 & $5 \cdot 1$ & 0.6 & $5 \cdot 3$ & 1.2 & $6 \cdot 6$ & 0.7 & 0.181 \\
\hline YCF† & $0^{\mathrm{a}}$ & 0 & $0^{\mathrm{a}}$ & 0 & $380^{\mathrm{b}}$ & 12 & $368^{\mathrm{b}}$ & 7 & $<0.001$ \\
\hline Breast milk & $32 \cdot 8^{\mathrm{a}}$ & $5 \cdot 6$ & $35 \cdot 9^{a}$ & $4 \cdot 7$ & $8 \cdot 6^{\mathrm{a}, \mathrm{b}}$ & 9.9 & $6 \cdot 3^{\mathrm{b}}$ & $5 \cdot 3$ & $<0.001$ \\
\hline \multicolumn{10}{|l|}{ CIF } \\
\hline Meat- and fish-based products and dishes & $0.0^{\mathrm{a}}$ & 0.0 & $34 \cdot 3^{\mathrm{b}}$ & $2 \cdot 3$ & $0.0^{\mathrm{a}}$ & 0.0 & $39 \cdot 3^{b}$ & 2.5 & $<0.001$ \\
\hline Other savoury-based foods and dishes & $0.0^{\mathrm{a}}$ & 0.0 & $9 \cdot 1^{\mathrm{b}}$ & 0.9 & $0.0^{\mathrm{a}}$ & 0.0 & $8.0^{\mathrm{b}}$ & 1.0 & $<0.001$ \\
\hline Fruit-based foods and dishes & $0.0^{\mathrm{a}}$ & 0.0 & $20 \cdot 4^{\mathrm{b}}$ & 1.7 & $0.0^{\mathrm{a}}$ & 0.0 & $25 \cdot 0^{\mathrm{b}}$ & 1.9 & $<0.001$ \\
\hline Dairy-based foods and dishes & $0.0^{\mathrm{a}}$ & 0.0 & $2 \cdot 5^{\mathrm{a}}$ & 0.7 & $0.0^{\mathrm{a}}$ & 0.0 & $6 \cdot 2^{b}$ & 0.8 & $<0.001$ \\
\hline Cereal-based foods and dishes & $0.0^{\mathrm{a}}$ & 0.0 & $8.9^{\mathrm{b}}$ & 1.3 & $0.0^{\mathrm{a}}$ & 0.0 & $16 \cdot 7^{\mathrm{c}}$ & $1 \cdot 4$ & $<0.001$ \\
\hline Snacks (sweet and savoury) & $0 \cdot 0^{\mathrm{a}}$ & 0.0 & $4 \cdot 0^{\mathrm{b}}$ & 0.2 & $0.0^{\mathrm{a}}$ & 0.0 & $4 \cdot 2^{b}$ & 0.3 & $<0.001$ \\
\hline Commercial infant beveragesf & 4.7 & 2.9 & $10 \cdot 7$ & 2.4 & 3.7 & $5 \cdot 1$ & $17 \cdot 8$ & $2 \cdot 7$ & 0.005 \\
\hline
\end{tabular}

YCF, young child formula; CIF, commercial infant foods; NMES, non-milk extrinsic sugars.

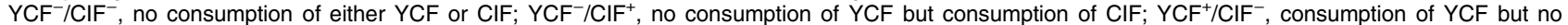
consumption of $\mathrm{CIF} ; \mathrm{YCF}^{+} / \mathrm{CIF}^{+}$, consumption of $\mathrm{YCF}$ and $\mathrm{CIF}$.

a,b,c Mean values within a row with unlike superscript letters were significantly different as tested by pair-wise post hoc comparisons, when ANCOVA identified a significant effect with Bonferroni correction $(P \leq 0.001)$.

${ }^{*}$ Adjusted for age and energy intake.

†In the total sample, the intakes of YCF consisted of $46 \%$ growing-up milk and $39 \%$ follow-on milk.

fln the total sample, the intakes of commercial infant beverages consisted mainly of fortified fruit juices.

probability of adequacy for vitamin $\mathrm{D}$ was low for all children, except for those in the fourth quartile of the PANDiet (0.65 (se 0.02)). Although our results are globally coherent with those from the DNSIYC study, a direct comparison of nutrient intakes should be made with caution given the expression of intakes is different (percentage of the RNI in DNSIYC and probability of adequacy in the PANDiet).

\section{Content and construct validity of the PANDiet}

We have confirmed that the PANDiet assesses nutrient adequacy independently of energy intake and that the children with the highest PANDiet score had a higher probability of adequacy for most of the nutrients. We also found that children with a higher PANDiet score were more likely to have food intakes in line with the dietary 
guidelines promoted by the UK health authorities by consuming less sweet foods and soft drinks, and more fruits and vegetables ${ }^{(4,5)}$. Finally, the associations we found between the PANDiet score and child and maternal characteristics were consistent with the literature. We found that children's diet quality was associated with having siblings ${ }^{(28)}$ and with having an older mother with a higher educational level ${ }^{(17,27)}$. The specific association we found with South Asian children (lower percentage in the lowest quartile of PANDiet) was not reported in the literature but could be explained by the lower intakes of $\mathrm{Na}$ and NMES of these children ${ }^{(19)}$. Our study provided strong evidence suggesting that the PANDiet is a valid tool to assess diet quality in young children. Due to the lack of a gold standard for estimating diet quality, it is not possible to validate a diet quality index using the classical criterion validation. Thus evaluating the content and construct validity is the most widely recognized approach to determine whether an index is valid. Because the PANDiet is a nutrient-based diet quality index, it could be easily applied to other countries or populations where relevant nutritional recommendations are available. We strongly recommend that the content and construct validity of any new versions of the PANDiet be evaluated.

\section{Diet quality and the consumption of young child formula and commercial infant foods}

According to the literature, the contribution of YCF in the diet of young children tends to be positive in terms of nutrient adequacy but negative in terms of dietary diversity. In French young children aged 12-24 months, the daily intake of YCF as a substitute for cow's milk reduced the risk of inadequacies of $\mathrm{Fe}, \alpha$-linolenic acid, vitamin $\mathrm{C}$ and vitamin $\mathrm{D}^{(9)}$, and in Irish young children aged 12-24 months, it reduced the risk of inadequacies of $\mathrm{Fe}$ and vitamin $\mathrm{D}^{(10)}$. Using a diet quality index measuring the total nutrient adequacy of the diet, it was found that German young children aged 10-36 months consuming YCF had a higher score than those who did not consume $\mathrm{YCF}^{(29)}$. In contrast, in Australian young children aged 12-16 months, a greater intake of YCF was associated with lower dietary diversity $^{(11)}$. In our study, the consumption of YCF was significantly associated with better nutritional adequacy of the diet but was not associated with a less diverse diet.

The literature concerning the contribution of CIF to the diet of young children is scarce and inconsistent. In terms of nutritional content, it was found that the UK CIF market mainly supplied products that are no more energy dense than YCF and much less nutrient dense than home-made foods ${ }^{(8)}$. In addition, US CIF (dinners, snacks, desserts and juice drinks) were of potential concern due to high $\mathrm{Na}$ or added sugar content ${ }^{(30)}$. In contrast, it was found that Spanish CIF have a higher energy density, a better ratio between the macronutrients and a similar salt content compared with UK home-prepared baby meals ${ }^{(12)}$. In terms of dietary pattern, it was found that vegetable variety was higher among children who consumed CIF compared with children who did not consume CIF in low-income US young children aged 6-12 months ${ }^{(13)}$. Moreover, children given CIF had a significantly higher vegetable variety than to those given home-made meals in German young children aged 12 months $^{(31)}$. In contrast, higher consumption of CIF was associated with lower vegetable intake in German young children aged 6-9 months but this association was no longer significant in pre-school and school-aged children ${ }^{(32)}$. Robinson et al. found two dietary patterns in UK young children aged 12 months, one characterized by a high consumption of fruit, vegetables and home-prepared foods and the other characterized by a high consumption of bread, savoury snacks, biscuits and chips, and both patterns characterized by a low frequency of consumption of $\mathrm{CIF}^{(27)}$. In our study, we found that the consumption of CIF was significantly associated with slightly better nutritional adequacy of the diet and was not associated with a lower probability of adequacy for NMES and $\mathrm{Na}$. In addition, the consumption of CIF was not associated with a less diverse diet. While PUFA intake was slightly but significantly lower in children consuming CIF, this is most probably related to the fact that CIF containing fish are still rare on the market. A wider range of CIF based on fish and combined fish/vegetable/potato options, along with more information about the advantages of fish consumption, might help to increase fish consumption and PUFA intake in toddlerhood and beyond. A recent scientific statement from the American Society for Nutrition concluded that for the US population (including young children aged more than 2 years), processed foods have intrinsic qualities (e.g. convenient, enjoyable, affordable) and contribute to both food and nutrition security. This statement also highlighted that diets are more likely to be healthy and balanced if nutrient-dense foods, either processed or not, are consumed ${ }^{(33)}$. Similarly, our results indicate that CIF could be used as part of a healthy balanced diet including a large variety of home-made baby foods. However, research regarding the variety in taste, flavour and texture, as well as the variety in the specific products is needed. Given the large variety of CIF available on the market, parents are advised to look carefully at the labels to ensure they select the healthier options $^{(4)}$.

\section{Strengths and limitations}

A major strength of our analysis is the use of the PANDiet which measures diet quality in terms of global nutrient adequacy rather than food intakes. As a result, we were able to objectively measure diet quality and to elucidate the contribution that YCF and CIF make to the nutrient adequacy of the diet. Any additional points to the PANDiet and its sub-scores are interpreted as preferable in terms of the nutrient adequacy of the diet. The application of the PANDiet to longitudinal data is needed to provide a more accurate interpretation of the effects of additional points 
on health. Another major strength of our study is the high-quality, nationally representative data and large sample that we used for the analyses.

Nevertheless, some important limitations should be considered when interpreting our findings. First, our results are based on an assessment of food consumption over four days, leading to a potential under- or overestimation of infrequently consumed foods. Also, food intake reporting was the full responsibility of the parent or caregiver, and it is not known to what extent there is risk for misreporting ${ }^{(19)}$. In order to minimize such risk, we excluded children identified as having extreme energy intakes.

Second, the nutrient intakes, and thus the nutritional adequacy, may have been over- or underestimated due to methodological issues. For example, food diaries are known to underestimate $\mathrm{Na}$ intake ${ }^{(34)}$. We anticipate this to be less of a problem in food prepared for young children, nevertheless it still requires consideration. Another issue is linked to differential variations in the nutrient content of home-made dishes and manufactured foods. CIF nutrient content might be more stable compared with home-prepared meals ${ }^{(12)}$, but results could quickly become outdated due to continuous introduction of new CIF and changes in formulation and production.

Another limitation is that our results are based on a selection of dietary recommendations. Because no UK nutritional recommendations for children aged less than 5 years were available for total fat, SFA, PUFA, total carbohydrates and NMES, we chose to use European Food Safety Authority recommendations for total fat, PUFA and total carbohydrates and the UK recommendations for children aged 5 years and over for NMES and SFA. In addition, the current UK nutritional recommendations do not integrate the recent growth standards which led to a consequent reduction in energy estimated average requirement ${ }^{(35)}$. We acknowledge that the construction of the PANDiet using different sets of recommendations is not ideal. However, we believe the recommendations we selected provide, as far as possible, a pertinent overview of the requirements for this population group.

Finally, defining consumers of CIF and YCF as those with an intake higher than $0 \mathrm{~g}$ prevented us from understanding the effect of the level of consumption of YCF and/or CIF on the nutritional adequacy of the diet. Nevertheless, such approach would increase the number of groups for the analysis, consequently decrease the number of participants per group and result in lack of power for statistical testing. Because the distribution of CIF intake was similar in the $\mathrm{YCF}^{-} / \mathrm{CIF}^{+}$and $\mathrm{YCF}^{+} / \mathrm{CIF}^{+}$ groups, as well as the distribution of $\mathrm{YCF}$ in the $\mathrm{YCF}^{+} / \mathrm{CIF}^{-}$ and $\mathrm{YCF}^{+} / \mathrm{CIF}^{+}$groups (data not shown), our results are not considered to be biased by different levels of consumption in the studied groups. Further analysis with greater numbers of participants is needed to better understand the nutritional adequacy of the diet at different levels of consumption of YCF and CIF.

\section{Conclusion}

In conclusion, the PANDiet is a valid indicator of the nutrient adequacy of the diet of UK young children. In this population, the specific consumption of CIF is not associated with lower nutritional adequacy of the diet, whereas the consumption of YCF is associated with a significantly higher probability of adequacy for several nutrients. In addition, the consumption of YCF and CIF is not associated with a difference in the consumption of fruits and vegetables. Our results indicate that young children who consume both YCF and CIF have an increased likelihood of meeting their nutrient recommendations. Further studies in this and other populations are needed to better understand different levels of consumption of YCF and CIF and the contribution they make to a healthy diet. Additionally, the consequences of the consumption of these products on the dietary and health patterns of children are areas requiring further investigation.

\section{Acknowledgements}

Acknowledgements: The authors gratefully acknowledge NatCen Social Research as main investigator and depositor, for access to the DNSIYC database and support documentation. The findings and conclusions of this study are those of the authors. Financial support: This study was supported by Danone Nutricia Research. No additional external funding was received for this study. Danone Nutricia Research had no role in the study design, data collection or in the decision to publish. Conflict of interest: B.A.H. and S.E. were employed by Danone Nutricia Research during the conduct of the study. Authorship: B.A.H. designed and managed the present study. E.O.V. contributed to the design of the study, carried out the statistical analysis and drafted the manuscript. E.O.V and B.A.H. interpreted the results. B.A.H. and S.E. contributed to writing the manuscript and provided critical comments. All of the authors read and approved the final manuscript. Ethics of human subject participation: DNSIYC was conducted according to the guidelines laid down in the Declaration of Helsinki and all procedures involving human subjects were approved by the Cambridgeshire 4 Research Ethics Committee on 18 January 2010. Written informed consent was obtained from all parents of the children taking part in DNSIYC.

\section{Supplementary material}

To view supplementary material for this article, please visit http://dx.doi.org/10.1017/S1368980015003134 


\section{References}

1. World Health Organization (2014) Infant and young child feeding. Fact sheet $\mathrm{N}^{\circ} 342$. http://www.who.int/ mediacentre/factsheets/fs342 (accessed March 2015).

2. Skinner JD, Ziegler P, Pac S et al. (2004) Meal and snack patterns of infants and toddlers. J Am Diet Assoc 104, Suppl. 1, S65-S70.

3. Carruth BR, Ziegler PJ, Gordon A et al. (2004) Developmental milestones and self-feeding behaviors in infants and toddlers. J Am Diet Assoc 104, Suppl. 1, S51-S56.

4. Department of Health (2014) Birth to five. http://www. publichealth.hscni.net/publications/birth-five (accessed March 2015)

5. British Nutrition Foundation (2014) 5532 a-day - perfect portions for toddler tums. http://www.nutrition.org.uk/ healthyliving/toddlers/5532.html (accessed March 2015).

6. EFSA Panel on Dietetic Products, Nutrition and Allergies (2013) Scientific Opinion on nutrient requirements and dietary intakes of infants and young children in the European Union. EFSA J 11, 3408.

7. Which? (2014) Toddler formula milk. http://www.which.co, uk/baby-and-child/nursery-and-feeding/guides/choosing-theright-formula-milk/toddler-formula-milk-/ (accessed March 2015).

8. García AL, Raza S, Parrett A et al. (2013) Nutritional content of infant commercial weaning foods in the UK. Arch Dis Child 98, 793-797.

9. Ghisolfi J, Fantino M, Turck D et al. (2013) Nutrient intakes of children aged 1-2 years as a function of milk consumption, cows' milk or growing-up milk. Public Health Nutr $\mathbf{1 6}$, 524-534.

10. Walton J \& Flynn A (2013) Nutritional adequacy of diets containing growing up milks or unfortified cow's milk in Irish children (aged 12-24 months). Food Nutr Res 57, 21836.

11. Byrne R, Magarey A \& Daniels L (2014) Food and beverage intake in Australian children aged 12-16 months participating in the NOURISH and SAIDI studies. Aust NZ J Public Health 38, 326-331.

12. Van den Boom S, Kimber AC \& Morgan JB (1997) Nutritional composition of home-prepared baby meals in Madrid. Comparison with commercial products in Spain and home-made meals in England. Acta Paediatr 86, $57-62$.

13. Hurley KM \& Black MM (2010) Commercial baby food consumption and dietary variety in a statewide sample of infants receiving benefits from the special supplemental nutrition program for women, infants, and children. $J \mathrm{Am}$ Diet Assoc 110, 1537-1541.

14. Waijers PMCM, Feskens EJM \& Ocké MC (2007) A critical review of predefined diet quality scores. Br J Nutr 97, 219-231.

15. Lazarou C \& Newby PK (2011) Use of dietary indexes among children in developed countries. Adv Nutr 2, 295-303.

16. Kyttälä P, Erkkola M, Lehtinen-Jacks S et al. (2014) Finnish Children Healthy Eating Index (FCHEI) and its associations with family and child characteristics in pre-school children. Public Health Nutr 17, 2519-2527.

17. Golley RK, Smithers LG, Mittinty MN et al. (2012) An index measuring adherence to complementary feeding guidelines has convergent validity as a measure of infant diet quality. J Nutr 142, 901-908.
18. Verger EO, Mariotti F, Holmes BA et al. (2012) Evaluation of a diet quality index based on the probability of adequate nutrient intake (PANDiet) using national French and US dietary surveys. PLoS One 7, e42155.

19. Lennox A, Sommerville J, Ong K et al. (2013) Diet and Nutrition Survey of Infants and Young Children, 2011. A Survey Carried out on Behalf of the Department of Health and Food Standards Agency. London: Department of Health.

20. Smithers G (1993) MAFF's nutrient databank. Nutr Food Sci 2, 16-19.

21. Howell DC (2008) Méthodes Statistiques en Science Humaines (Statistical Methods in Human Sciences). Louvain-la-Neuve: De Boeck Université.

22. Kelly SA, Summerbell C, Rugg-Gunn AJ et al. (2005) Comparison of methods to estimate non-milk extrinsic sugars and their application to sugars in the diet of young adolescents. Br J Nutr 94, 114-124.

23. Department of Health (1991) Dietary Reference Values for Food Energy and Nutrients for the United Kingdom. Report on Health and Social Subjects no. 41. London: HM Stationery Office.

24. Nordic Council of Ministers (2014) Nordic Nutrition Recommendations 2012: Integrating nutrition and physical activity. http://dx.doi.org/10.6027/Nord2014-002 (accessed March 2015).

25. Smithers LG, Golley RK, Brazionis L et al. (2011) Characterizing whole diets of young children from developed countries and the association between diet and health: a systematic review. Nutr Rev 69, 449-467.

26. Marshall S, Burrows T \& Collins CE (2014) Systematic review of diet quality indices and their associations with health-related outcomes in children and adolescents. J Hum Nutr Diet 27, 577-598.

27. Robinson S, Marriott L, Poole J et al. (2007) Dietary patterns in infancy: the importance of maternal and family influences on feeding practice. Br J Nutr 98, 1029-1037.

28. Patrick H \& Nicklas TA (2005) A review of family and social determinants of children's eating patterns and diet quality. J Am Coll Nutr 24, 83-92.

29. Hilbig A, Drossard C, Kersting M et al. (2015) Nutrient adequacy and associated factors in a nationwide sample of German toddlers. J Pediatr Gastroenterol Nutr 61, 130-137.

30. Cogswell ME, Gunn JP, Yuan K et al. (2015) Sodium and sugar in complementary infant and toddler foods sold in the United States. Pediatrics 135, 416-423.

31. Mesch CM, Stimming M, Foterek K et al. (2014) Food variety in commercial and homemade complementary meals for infants in Germany. Market survey and dietary practice. Appetite 76, 113-119.

32. Foterek K, Hilbig A \& Alexy U (2015) Associations between commercial complementary food consumption and fruit and vegetable intake in children. Results of the DONALD study. Appetite 85, 84-90.

33. Weaver CM, Dwyer J, Fulgoni VL et al. (2014) Processed foods: contributions to nutrition. Am J Clin Nutr 99, $1525-1542$.

34. Brown IJ, Tzoulaki I, Candeias V et al. (2009) Salt intakes around the world: implications for public health. Int $J$ Epidemiol 38, 791-813.

35. Scientific Advisory Committee on Nutrition (2007) SACN Application of WHO Growth Standards in the UK. https:// www.gov.uk/government/publications/sacn-application-ofwho-growth-standards-in-the-uk (accessed March 2015). 